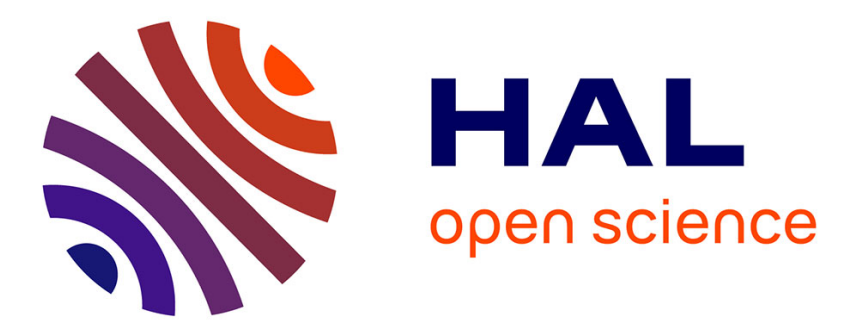

\title{
L'apparition des meules rotatives en Languedoc oriental (IVe s. avant J.-C.) d'après l'étude du site de Lattes
} Jean-Louis Reille

\section{To cite this version:}

Jean-Louis Reille. L'apparition des meules rotatives en Languedoc oriental (IVe s. avant J.-C.) d'après l'étude du site de Lattes. Gallia - Archéologie de la France antique, 2000, 57, pp.261-272. 10.3406/galia.2000.3022 . hal-01902072

\section{HAL Id: hal-01902072 \\ https://hal.science/hal-01902072}

Submitted on 16 Jan 2020

HAL is a multi-disciplinary open access archive for the deposit and dissemination of scientific research documents, whether they are published or not. The documents may come from teaching and research institutions in France or abroad, or from public or private research centers.
L'archive ouverte pluridisciplinaire HAL, est destinée au dépôt et à la diffusion de documents scientifiques de niveau recherche, publiés ou non, émanant des établissements d'enseignement et de recherche français ou étrangers, des laboratoires publics ou privés.

\section{(ㅇ)(1) $\$$}

Distributed under a Creative Commons Attribution - NonCommercial - NoDerivatives $\mid 4.0$ 


\title{
L'APPARITION DES MEULES ROTATIVES EN LANGUEDOC ORIENTAL (IV S. AVANT J.-C.) D’APRÈS L'ÉTUDE DU SITE DE LATTES
}

\author{
Jean-Louis REILLE*
}

\begin{abstract}
Mots-clés. Protohistoire, Languedoc oriental, meules rotatives, basalte, analyse pétrographique, détermination des provenances, sites de production, sources du matériel lithique, diffusion spatiale.
\end{abstract}

Key-words. Protohistory, eastern Languedoc, rotary millstones, basalt, petrographic analysis, determination of origin, production sites, sources of lithic material, spatial distribution.

Résumé. L'analyse pétrographique microtexturale appliquée aux meules basaltiques du site portuaire de Lattes (Languedoc oriental) a permis de localiser les lieux d'extraction de ce mobilier lithique, au IVE s. avani J.-C. À cette époque, le plus important d'entre eux se trouvait dans l'arrière-pays d'Agde à proximité du volcan de Saint-Thibéry/Bessan, dans la basse vallée de l'Hérault. Ce centre d'extraction n'est pas attesté in situ par des vestiges archéologiques; il est connu seulement par sa production. Dans l'état actuel des données, il a fourni les plus anciens spécimens de meules rolatives actuellement découverts en Languedoc oriental et en Provence (premier quart du IV $V^{p}$. avant J.-C.). Plusieurs arguments permettent de le considérer comme un centre enraciné dans la tradition technique ibéro-languedocienne. Toutefois, situé aux limites du territoire de la colonie grecque d'Agde (fondée à la fin du Ves.), il a probablement été intégré assez tôt à l'activité commerciale maritime et terrestre de cette cité, au bénéfice de la diffusion de ses produits. Comparativement, à cette époque, l'activité du célèbre site d'Émbonne/Cap d'Agde paraît se situer à l'arrière-plan, à l'inverse de ce que l'on observe aux Ir et Ir s. avant J.-C.

Abstract. Petrographic analysis was applied to $4^{\text {th }}$ century BC millstones from the Protohistoric lagoonal harbour of Lalles (soulhern France, near Montpellier), in order to locate their origin. The most important quarrying centre was located in the lower valley of Hérault river, near the basaltic outcrops of Saint-Thibéry/Bessan. There are no archaeological field traces about this hypothetic worksite, only known through its products. According to present data, it furnished the oldest rotary millstones excavated in eastern Languedoc and Provence (first quarter of $4^{\text {th }}$ century BC). From several facts, one may suppose that its production takes roots in the Iberic local technical culture. Nevertheless, it was probably early integrated into the big commercial activity of the Greek colony of Agde. Comparatively, during this period, the famous quarries of "Embonne/Cap d'Agde " seem to be commercially relegated to the background.

En France méditerranéenne, les gisements de laves basaltiques sont en nombre limité et étroitement localisés. Or, dans ce vaste territoire, le mobilier archéologique de l'âge du Fer et de l'Antiquité comporte une proportion élevée de meules en basalte. Cette ubiquité, que l'on observe sur une durée de près de dix siècles, atteste l'importance des échanges relatifs à ces objets de la vie quotidienne et l'intérêt que l'on peut attacher à la détermination de leur provenance.

* UMR 154 du CNRS, université Montpellier II Sciences, case courrier 057, F-34095 Montpellier cedex 5. Mél : reille@dstu.univ-montp2.fr 
Nous avons montré précédemment, à propos de quelques sites archéologiques, comment l'analyse pétrographique microtexturale en lames minces permet d'identifier les principales sources du matériau constitutif des meules et d'ébaucher la reconstitution de leurs trajectoires de distribution à l'échelon régional (Reille, 1995, 1998, 1999).

Sans entrer dans le détail de la démarche, antérieurement développée (Reille, 1995, annexe I), il nous paraît utile d'en résumer ici l'essentiel.

Dans un premier temps, on recherche systématiquement si les divers gisements de roches basaltiques $(s . l$.) possèdent des caractéristiques microtexturales suffisamment originales permettant de les distinguer. À partir de ces données, on élabore un catalogue de référence ouvert, extensible au gré des investigations complémentaires.

L'étape suivante consiste à comparer les microtextures observées sur les meules avec celles répertoriées dans le référentiel, afin de se prononcer sur l'origine probable du matériau en cas d'analogies particulièrement évidentes.

Les conclusions auxquelles on aboutit en fin d'observation peuvent se classer en trois catégories.

$1^{\text {re }}$ catégorie : la microtexture observée dans l'objet ne peut être rattachée à aucune microtexture du référentiel : l'origine est alors, en principe, strictement indéterminée. Toutefois, dans le cas spécifique où le type de la roche volcanique n'est pas connu en France, on peut conclure à une origine exotique. Pour la Gaule méridionale on est souvent renvoyé vers le monde méditerranéen (Italie continentale, Sardaigne, Sicile, etc.).

Jusqu'à ce jour, dans notre pratique, la proportion des cas relevant de cette première catégorie est relativement peu élevée.

$2^{\text {e }}$ catégorie : la microtexture observée dans l'objet correspond, dans le référentiel, à celle d'un gisement important, archéologiquement reconnu pour avoir abrité un ou plusieurs sites d'extraction de meules. Tel est le cas de sites éminents comme Le Cap d'Agde en Languedoc et ceux du secteur de La Courtine, près de Toulon. C'est la catégorie la plus courante dans nos investigations antérieures (plus de $80 \%$ du matériel examiné).

Inversement, la question de l'utilisation éventuelle d'un gisement non attesté archéologiquement mais soupçonné d'avoir alimenté le commerce des meules peut recevoir un début de réponse. C'est le cas pour

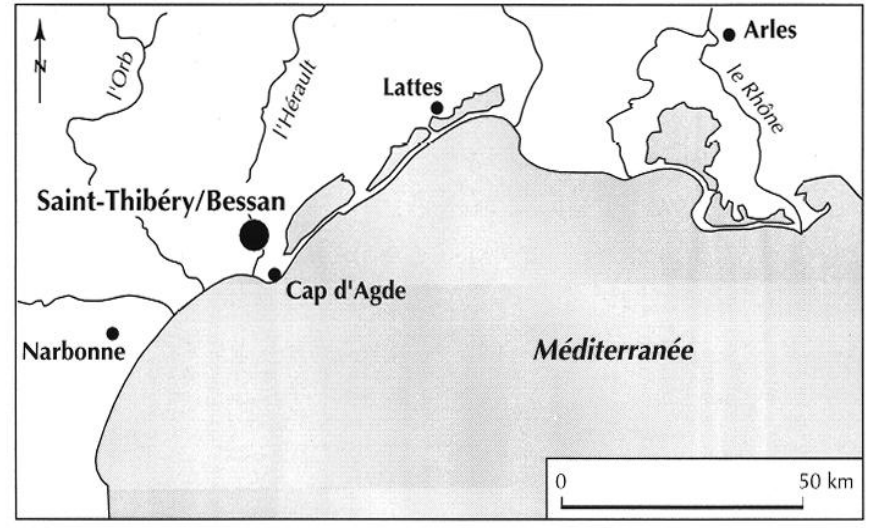

Fig. 1 - Situation géographique du porl lagunaire de Lattes par rapport aux gisements de basalte du Cap d'Agde el de SaintThibéry/Bessan.

l'ensemble basaltique de Beaulieu, à proximité d'Aix-enProvence. En effet, contrairement à ce que l'on aurait pu attendre, les caractéristiques microtexturales distinctives de ses laves n'ont jamais été observées (au moins jusquelà) parmi les nombreux éléments de meules du II' ou du $\mathrm{I}^{\mathrm{er}} \mathrm{s}$. avant J.-C. exhumés dans la ville d'Aix ou l'oppidum d'Entremont (Reille, 2000b).

$3^{\text {e }}$ catégorie: on retrouve, dans le matériau des meules, sur une durée et avec une fréquence significatives, la signature spécifique d'un gisement individualisé dans le référentiel microtextural mais non attesté comme site d'extraction sur le plan archéologique. Sur le plan méthodologique, cette dernière catégorie de conclusions est spécialement intéressante, notamment par le type de questions qu'elle peut susciter.

À titre d'exemple, nous nous proposons de présenter et de commenter ici plusieurs résultats analytiques qui relèvent de la troisième catégorie. Ils ont été obtenus sur du matériel du IV ${ }^{e}$ s. avant J.-C., et nous renvoient systématiquement vers le complexe volcanique dit " de SaintThibéry ", plus précisément situé sur le territoire des communes de Saint-Thibéry et de Bessan dans la basse vallée de l'Hérault (fig. 1). En effet, le gisement basaltique de Saint-Thibéry/Bessan n'est pas archéologiquement attesté comme site d'extraction de meules, alors qu'on l'identifie fréquemment en tant que source de ce mobilier, notamment en Languedoc oriental, au $\mathrm{IV}^{\mathrm{re}} \mathrm{s}$. avant J.-C. De plus, les meules qui en proviennent semblent avoir joué un rôle significatif dans la diffusion des premiers systèmes rotatifs dans cette partie de la Gaule méridionale. 
Nous savons que les modalités d'apparition du modèle rotatif intéressent spécialement l'histoire des techniques, car il s'agit d'une innovation importante par rapport aux anciens systèmes de mouture qu'il tinira par supplanter (meules archaïques à va-et-vient et meules à trémie de type grec). Pour ces questions, nous renvoyons le lecteur vers des publications récentes concernant le sujet, spécialement celles de N. Alonso-Martinez, H. Amouric, J.-P. Brun, C. Domergue et al., que l'on trouvera réunies dans un volume édité par D. Garcia et D. Meeks (1997). D'autre part, une revue complète de la question est présentée dans l'ouvrage de N. AlonsoMartinez (1999, p. 231-269). On rappellera ici que les premières meules rotatives basses du bassin méditerranéen apparaissent sur la façade nord-orientale de l'Ibérie, spécialement en Catalogne, dans le courant du $V$ 's. avant J-C. Leur diffusion s'effectue d'abord vers le nord, en Languedoc occidental, ensuite d'ouest en est vers le Languedoc oriental, puis la Provence (Py, 1992 ; Alonso-Martinez, 1997, 1999). Dans ce contexte, les données concernant l'époque et les modalités d'apparition de ce système de mouture en Languedoc oriental présentent quelque intérêt.

Dans notre étude, le terme « rotatif » est appliqué indifféremment à toute meule circulaire à mouvement rotatif ou semi-rotatif. Sur le plan historique, l'évaluation des rôles respectifs des gisements basaltiques du Cap d'Agde et de Saint-Thibéry/Bessan dans la diffusion des meules au IV s. avant J.-C., en Languedoc oriental, contraste singulièrement avec ce que l'on observe à la fin de l'âge du Fer.

\section{LES BASALTES DE SAINT-THIBÉRY/BESSAN DANS LE VOLCANISME DE LA BASSE VALLÉE DE L'HÉRAULT}

Dans la basse vallée de l'Hérault, les affleurements de lave les plus proches du littoral se rencontrent sous l'actuelle ville d'Agde et ses abords; on en trouve aussi sur la côte au niveau du Cap d'Agde et de Rochelongue/ Grau d'Agde (fig. 2). Dans l'ensemble de ce secteur où la superficie des affleurements de lave n'excède pas $6 \mathrm{~km}^{2}$, on peut néanmoins caractériser, sur le plan textural, trois microfaciès distincts parmi les roches basaltiques.

Le premier authentifie le basalte alcalin d'Embonne/ Cap d'Agde, qui a joué pendant plus de trois siècles un

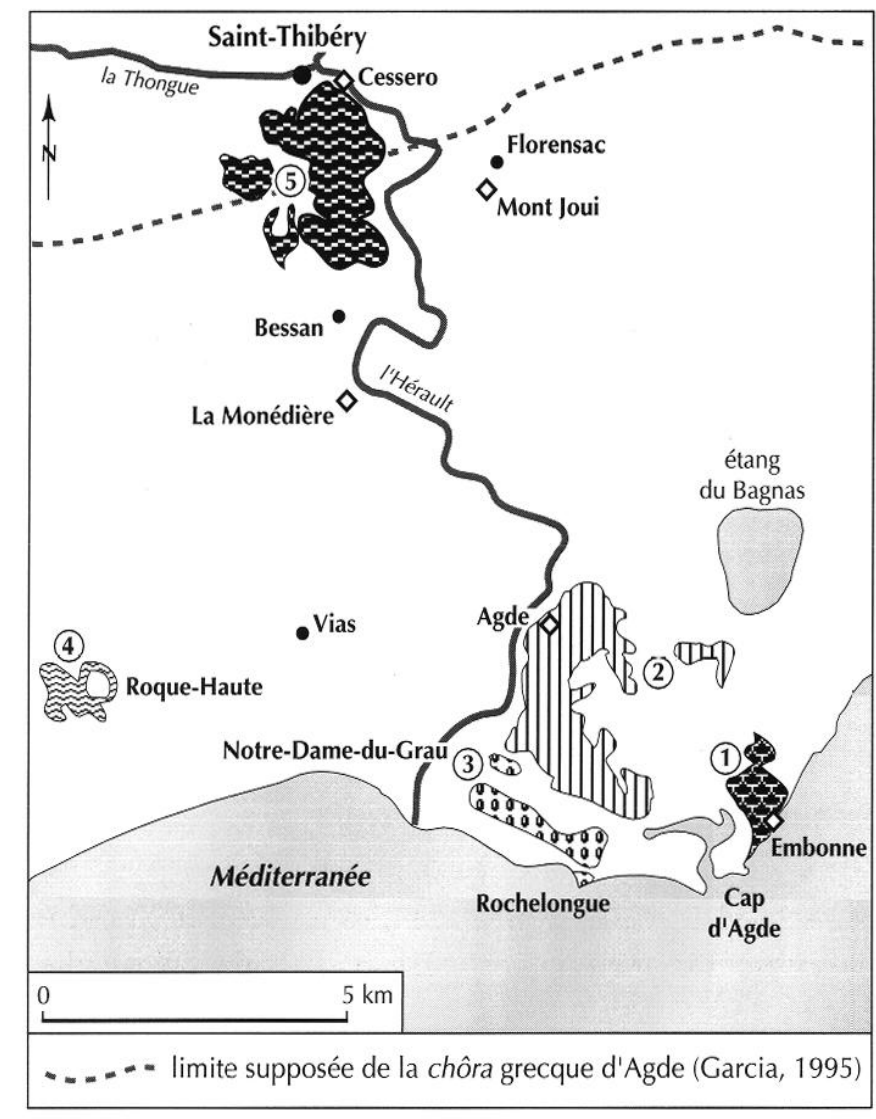

Fig. 2 - Répartition des principaux affleurements de laves basaltiques dans les environs de la cité d'Agde et la basse vallée de l'Héraull; d'après les données de la carte géologique de la France à l'échelle de $1 / 50000$ (feuille d'Agde, $n^{\circ} 1015$ ), modifiée. Les chiffres de. 1 à 5 correspondent aux différents types microtexturaux identifiés dans les roches basaltiques; une appréciation est donnée ci-dessous sur leur utilisation pour la confection de meules, toutes formes confondues : 1, basalte alcalin du Cap d'Agde largement exploité à partir du II"s. avant J.-C. (utilisation modérée au IVes. avant J.-C.) ; 2, basalte alcalin d'Agde-ville (pas d'utilisation attestée à ce jour) ; 3, basalte transitionnel de Notre-Dame-du-Grau et de Rochelongue/Grau d'Agde (quelques exemples d'utilisation antérieurement au II' s. avant J.-C.) ; 4, basalle transitionnel de Roque-Haute (pas d'utilisation attestée à ce jour) ; 5, basalte alcalin de Saint-Thibéry/Bessan (couramment utilisé pendant le $I^{E} s$. avant J.-C., son emploi pour la confection de meules se poursuit pendant plus de trois siècles). Les affleurements de tufs pyroclastiques ne sont pas représentés.

rôle de premier plan dans la fabrication des meules, notamment rotatives, ceci à une échelle que l'on pourrait qualifier d'industrielle.

Les deux autres microfaciès correspondent au basalte alcalin d'Agde-ville et au basalte " transitionnel " de 
Rochelongue/Grau d'Agde. Dans l'état actuel des connaissances, le basalte de Rochelongue/Grau d'Agde a été rarement utilisé (quelques meules à va-et-vient) ; quant au basalte d'Agde-ville il semble ne jamais avoir servi à la fabrication de meules, quel qu'en soit le modèle. Comme rien dans les propriétés mécaniques de ces roches ne limitait ou n'interdisait un tel usage, il faut chercher ailleurs la cause de cette désaffection.

Le volcan de Saint-Thibéry/Bessan est situé dans la basse vallée de l'Hérault, à $8 \mathrm{~km}$ au nord-nord-ouest de la ville d'Agde, $12 \mathrm{~km}$ du Cap d'Agde et $9 \mathrm{~km}$ du cordon littoral le plus proche (fig. 2). Par rapport aux sites de la périphérie agathoise et compte tenu des modalités de transport à l'époque qui nous intéresse, c'est incontestablement un site de l'arrière-pays.

Du point de vue géochimique, le basalte alcalin de Saint-Thibéry/Bessan est tout à fait analogue aux basaltes alcalins du Cap d'Agde et d'Agde-ville (Lefèvre, Dupuy, 1972 ; Dautria, Liotard, 1990).

En revanche, du point de vue microtextural, on peut le distinguer sans ambiguité des laves du secteur d'Agde (Reille, 1995, annexe III).

Aujourd'hui, comme dans un passé récent, le secteur de Saint-Thibéry/Bessan est un site de carrières intensément et extensivement exploitées en vue de l'extraction de blocs rocheux bruts et de la fabrication de granulats. Vu l'importance des ablations et décapages qui s'y succèdent, la probabilité d'y retrouver des vestiges d'extractions antiques s'amenuise de jour en jour.

\section{L'ANCIEN PORT LAGUNAIRE DE LATTES, TÉMOIN DE L'ÉVOLUTION DU COMMERCE CÔTIER AU SECOND ÂGE DU FER}

En l'absence d'archives, le seul moyen d'appréhender l'activité exportatrice d'un site aujourd'hui disparu consiste à décompter, sur d'autres sites, les importations qui en sont issues.

Pour le problème abordé ici, le site archéologique portuaire de Lattes présente un double intérêt.

Fondé dans la seconde moitié du Vle s. avant J.-C., il est précocement ouvert aux influences étrusques et helléniques. D'autre part, sa proximité relative par rapport à Agde et à la basse vallée de l'Hérault en fait un réceptacle de choix pour les exportations de leurs produits. C'est spécialement vrai pour les meules, car les matériaux rocheux font complètement défaut dans la plaine côtière de Lattes.

Déclaré d'intérêt national depuis 1995, le site archéologique de Lattes fait l'objet de fouilles programmées depuis 1983 (Py, Garcia, 1993). On dispose donc sur place d'un matériel d'étude soigneusement répertorié, abondant, et bien repéré sur le plan stratigraphique.

\section{LES EXPORTATIONS DE MEULES DU CAP D'AGDE VERS LATTES ET LA PROVENCE,} AUX II $^{\mathrm{e}}$ ET I ${ }^{\mathrm{er}}$ S. AVANT J.-C.

On sait qu'aux $\mathrm{II}^{\mathrm{e}}$ et $\mathrm{I}^{\mathrm{er}}$ s. avant J.-C., les meules de Lattes étaient quasi exclusivement taillées dans le basalte du Cap d'Agde (Dautria, Reille, 1992) et qu'elles appartiennent essentiellement au type rotatif (type B2, cf. Py, 1992) ou plus accessoirement à trémie (type A3, cf. ibid.). À cette époque, en effet, les types archaiques à va-et-vient (types $\mathrm{Al}$ et $\mathrm{A} 2, c f . i b i d$.) avaient pratiquement été abandonnés au profit d'instruments plus performants.

Pendant la même période, ce quasi-monopole du Cap d'Agde dans la fourniture des meules (souvent plus de $80 \%)$ se retrouve aussi bien dans le proche environnement de Marseille (Reille, Chabot, 2000) que dans les sites provençaux majeurs du secteur de Martigues, l'oppidum salyen d'Entremont, ainsi qu'à Aix-ville (musée Granet, 1987 ; Nin, 1991 ; Chausserie-Laprée, 1998 ; Reille, 1998, 2000b).

\section{LES IMPORTATIONS DE MEULES À LATTES, AU IV S. AVANT J.-C. ET LE RÔLE DE SAINT- THIBÉRY/BESSAN}

Une incursion dans des niveaux stratigraphiquement plus anciens révèle un tableau bien différent du précédent. C'est dans ces niveaux que l'on rencontre les premières meules rotatives actuellement connues. Nettement plus rares qu'aux époques postérieures, elles cohabitent avec nombre de meules traditionnelles du type à va-et-vient (fig. 4). Elles sont aussi beaucoup plus massives, caractère que l'on retrouve fréquemment dans les modèles rotatifs archaiques (Jannoray, 1955 ; AlonsoMartinez, 1999). Enfin, aucun exemplaire ou fragment 
d'exemplaire « grec » à trémie n'a été exhumé jusqu'à ce jour dans les niveaux lattois du IV $s$.

Tous échantillons confondus (70 exemplaires), l'analyse microtexturale montre d'abord que le matériel volcanique du Cap d'Agde joue un rôle tout à fait mineur par rapport au matériel issu d'autres gisements. Il ne représente, en effet, que $14 \%$ du total, et ce résultat est inattendu.

Parmi les autres sources, certaines sont méditerranéennes «exotiques », d'autres sont à rattacher à la moyenne vallée de l'Hérault. Enfin, quelques exemplaires non basaltiques ont vraisemblablement une origine plus occidentale (Reille, 1999).

Parmi les basaltes littoraux ou proches du littoral (fig. 2), seul celui d'Embonne/Cap d'Agde joue un rôle appréciable. Ceux d'Agde-ville et Roque-Haute (près de Vias) ne sont pas représentés. Celui de Rochelongue/ Grau d'Agde l'est fort peu. À l'inverse, le basalte de SaintThibéry/Bessan est largement utilisé, et il est intéressant de comparer son emploi avec celui du Cap d'Agde.

\section{RÔLES COMPARÉS DES BASALTES DU CAP D'AGDE ET DE SAINT-THIBÉRY/BESSAN DANS LES IMPORTATIONS LATTOISES DU IV ${ }^{\mathrm{e}} \mathrm{S}$. AVANT J.-C.}

Dans cette partie de l'étude, la population retenue a été restreinte aux seuls échantillons portant la signature microtexturale du Cap d'Agde ou de Saint-Thibéry/ Bessan (Reille, 1995).

Les échantillons de meules examinés à Lattes sont généralement fragmentaires. On peut les classer en trois catégories: ceux qui proviennent sans ambiguité de meules rotatives (catilli ou meta), ceux qui proviennent sans ambiguité de meules à va-et-vient (molettes ou tables), enfin ceux trop mal conservés pour être rattachés à l'un ou l'autre type (fig. 3).

Pour les meules rotatives, au nombre de huit, les proportions respectives sont de $25 \%$ pour Cap d'Agde et $75 \%$ pour Saint-Thibéry/Bessan. Pour les meules à va-etvient (total 27), on décompte $11 \%$ pour Cap d'Agde et $89 \%$ pour Saint-Thibéry/Bessan. Pour les fragments informes (total 14), on dénombre $35 \%$ pour Cap d'Agde et $65 \%$ pour Saint-Thibéry/Bessan.

Pour l'ensemble des échantillons retenus (total 49), les proportions sont de $20 \%$ pour Cap d'Agde et $80 \%$ pour Saint-Thibéry/Bessan, soit un rapport de un à

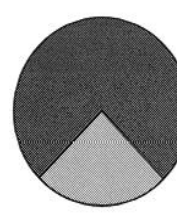

8 rotatives

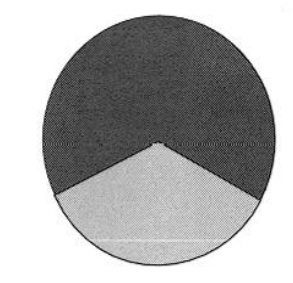

14 fragments

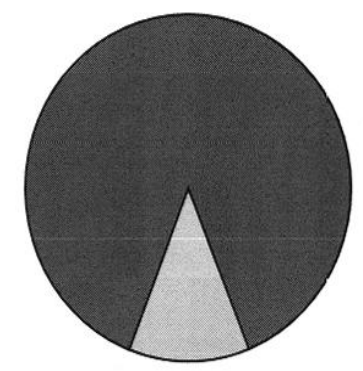

27 va-et-vient

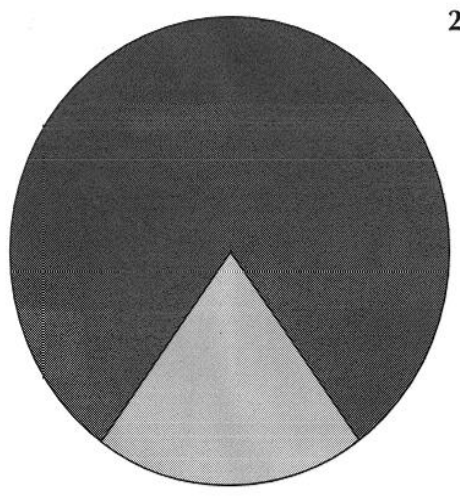

population totale : 49 échantillons

Saint-Thibéry/Bessan

Cap d'Agde

Fig. 3 - Rôles respectifs du basalte de Saint-Thibéry/Bessan et du basalte du Cap d'Agde en tant que sources du malériel lithique pour les meules de Lattes au $V^{e}$ s. avant J.-C. La surface des cercles est proportionnelle au nombre d'échantillons. La population traitée ici est réduite au sous-ensemble des échantillons dont la lave provient soit du Cap d'Agde soit de Saint-Thibéry/Bessan; elle représente les trois quarts de la population totale, toutes provenances confondues.

quatre. Cette disproportion permanente et particulièrement marquée pose à la fois la question de l'existence d'un centre de fabrication de meules dans les environs des affleurements volcaniques de Saint-Thibéry/Bessan et celle de son importance par comparaison avec le célèbre gisement d'Embonne-Cap d'Agde tel qu'il se présentait au IV ${ }^{\mathrm{e}} \mathrm{s}$. avant J.-C.

\section{UN CENTRE DE FABRICATION DE MEULES PROCHE DU VOLCAN DE SAINT-THIBÉRY/BESSAN ?}

L'étude archéologique de plusieurs carrières de meules antiques montre que des ébauches de ces outils étaient réalisées sur les sites d'extraction du matériau 
brut ou à proximité immédiate de ceux-ci (Bottin, 1905 ; Layet, 1950 ; Peacock, 1986).

Pour le gisement de Saint-Thibéry/Bessan on ne dispose pas, actuellement, d'indices archéologiques attestant une telle activité. On peut même craindre qu'ils aient disparu au cours des dernières décennies, par suite de l'intense activité des carrières. Un habitat protohistorique indigène est certes connu à Saint-Thibéry, sur le site de Cessero, du VI ${ }^{\mathrm{e}}$ au V ${ }^{\mathrm{re}} \mathrm{s}$. avant J.-C. (Coulouma, Claustres, 1943 ; Jully, 1983), mais il paraît abandonné ultérieurement jusqu'à une réoccupation tardive au ${ }^{\mathrm{Ir}} \mathbf{s}$. avant J.-C. Il est toutefois possible que ces recherches n'aient concerné qu'une partie de l'ensemble du site protohistorique de Saint-Thibéry. À Bessan, l'important site de La Monédière, fouillé par André Nickels et son équipe, fonctionne essentiellement du début du VIe à la fin du V'c s. avant J.-C. (Nickels, 1989). Malheureusement, les meules qui y auraient été exhumées paraissent introuvables, tout comme pour le site voisin du Mont Joui à Florensac, occupé pendant la seconde moitié du $\mathrm{VI}^{\mathrm{e}} \mathrm{s}$. et le premier quart du Ve $\mathrm{s}$. avant J.-C. (Nickels, 1987).

En dépit de ces pièces manquantes, on n'adoptera pas la position rigoriste exprimée par l'équation " pas de vestiges = pas de site de fabrication $"$. On admettra donc l'existence d'un « centre » de fabrication exploitant les basaltes de Saint-Thibéry/Bessan, centre hypothétique mais dont les produits finis sont bien réels. Dans ce qui suit, le mot "centre " correspond à une entité économique définie uniquement par la production de meules à proximité de l'ensemble volcanique de Saint-Thibéry/Bessan, en vue de leur exportation. Rien n'interdit qu'il fût composé d'unités spatialement dispersées. Si un tel centre a effectivement existé, quel pouvait être son statut par rapport à la cité grecque d'Agde?

\section{SAINT-THIBÉRY/BESSAN : CENTRE « GREC » OU « INDIGÈNE * ?}

Le développement qui suit comporte deux parties : - la première tente de réunir les éléments factuels et objectifs dont on dispose actuellement afin de les verser au débat concernant la diffusion des meules rotatives dans le sud-est de la Gaule,

- la seconde, essentiellement spéculative, suggère une interprétation possible (et vraisemblablement provi- soire) des faits précédents, et se propose d'examiner l'hypothèse d'un centre de fabrication à connotation culturelle essentiellement indigène, intégré aux circuits commerciaux de la jeune colonie agathoise.

\section{ÉTAT ACTUEL DES DONNÉES}

Dans les sociétés indigènes du midi de la Gaule, la fabrication des meules en basalte (non rotatives) et leur diffusion vers des sites éloignés des gisementssources sont indubitablement antérieures à l'arrivée des Grecs. À Martigues, les meules basaltiques extraites du chenal de Caronte en association avec du mobilier du Bronze final en fournissent un exemple (ChausserieLaprée, 1998).

Les meules rotatives portant la signature microtexturale de Saint-Thibéry/Bessan sont exportées pendant au moins trois siècles. On les rencontre dès le IV $^{e}$ s. avant J.-C. à Lattes et dans la moyenne vallée de l'Hérault (La Ramasse); on les retrouve, de manière discrète mais indubitable, à Martigues et Aix-en-Provence aux II et I $^{\mathrm{er}}$ s. avant J.-C.

À Lattes, dans les niveaux du IV ${ }^{c}$ s. avant J.-C., les vestiges de meules et les fragments de basalte de SaintThibéry/Bessan sont quatre fois plus nombreux que ceux du Cap d'Agde. Quand on considère la prépondérance écrasante des produits du Cap aux époques tardives, cette inversion de tendance est frappante. Cette remarque est spécialement valable pour les meules rotatives.

Tous les éléments de meules basaltiques à trémie (type grec) que nous avons étudiés, tant à Lattes qu'à Martigues, Aix ou Entremont, sont exclusivement taillés dans le basalte du Cap d'Agde '. Aucun spécimen, sur plus de vingt-cinq examinés, ne porte la signature de Saint-Thibéry/Bessan.

À ce jour, aucun élément de meule à trémie (type grec) n'a été trouvé à Lattes dans des niveaux du $\mathrm{I}^{\mathrm{re}} \mathrm{s}$. avant J.-C.

À l'inverse, les meules rotatives les plus anciennes actuellement connues en Languedoc oriental ont été trouvées à Lattes dans des niveaux datés du premier

1. Fourchettes chronologiques des sites: Martigues ( $\mathrm{V}^{\mathrm{e}} \mathrm{s}$. avant J.-C./Antiquité) ; Aix-ville/terrain Coq ( ${ }^{\text {er }} \mathrm{s}$. avant J.-C.) ; Entremont (II ${ }^{\mathrm{c}} \mathrm{s}$. avant J-C.). À Lattes, les niveaux actuellement étudiés en stratigraphie vont du tout début du IV"s. avant J.-C. au I'r s. après J.-C. 
quart du IV e s. avant J.-C. ${ }^{2}$. Toutes sont taillées dans le basalte de Saint-Thibéry/Bessan. En stratigraphie, toujours selon les données actuelles, les plus anciens éléments de meules rotatives taillées dans le basalte du Cap d'Agde ne se rencontrent à Lattes qu'un demi-siècle plus tard, dans le troisième quart du IV $\mathrm{IV}^{\mathrm{e}} \mathrm{s}$. avant J.-C.

Parmi les très nombreuses meules exportées pendant plusieurs siècles à partir des ateliers du littoral agathois et de la basse vallée de l'Hérault, il faut noter l'absence permanente et tout à fait remarquable du basalte sur lequel s'élevait la totalité de la cité grecque d'Agde! Or les qualités intrinsèques du matériau basaltique sont hors de cause dans cette désaffection.

Aux époques protohistoriques tardives, on perçoit clairement l'emprise de Marseille grecque et de ses dépendances sur le commerce côtier. On a vu en effet que la diffusion des meules en Languedoc oriental et en Provence est très largement dominée par les productions du Cap d'Agde (Reille, 1998, 2000b), productions parmi lesquelles figurent nombre d'exemplaires à trémie, de type grec. On n'y trouve aucun exemplaire taillé dans le basalte d'Agde-ville, non plus que dans celui de Rochelongue/Grau d'Agde, pourtant représenté dans les exportations des temps anciens (fig. 2). Curieusement, toutefois, on rencontre dans les exportations tardives quelques meules (par ailleurs exclusivement rotatives) dont le basalte provient de l'arrière-pays, et plus précisément de Saint-Thibéry/Bessan (fig. 2).

\section{INTERPRÉTATIONS POSSIBLES}

Rappelons d'abord, à toutes fins utiles, que la création de la colonie grecque d'Agde est stratigraphiquement datée de la fin du V $\mathrm{V}^{\mathrm{e}}$ s. avant J.-C. (Nickels, 1976, 1995).

Les affleurements basaltiques de Saint-Thibéry/ Bessan ont la particularité de se trouver à cheval sur la limite géographique septentrionale de la chôra grecque d'Agde telle que la définit D. Garcia (1995b, fig. 4) (cf. fig. 2).

Les discussions concernant la position exacte de cette limite ou ses fluctuations dans le temps ne relèvent pas de notre compétence, et l'analyse pétrographique ne peut évidemment rien nous apprendre sur le statut du centre de Saint-Thibéry/Bessan par rapport à la colonie

2. Références : US 27253 , US 50207, US 24013. agathoise. Aussi, comme nous l'avons déjà dit, cette partie du développement est-elle essentiellement spéculative.

\section{Un centre directement exploité par les Grecs?}

Un coup d'œil sur la figure 2 met en évidence l'aspect apparemment paradoxal de cette hypothèse. On s'explique mal pourquoi l'entreprise des colons grecs aurait débuté aux confins septentrionaux de leur nouveau territoire sur les gisements de basalte les plus éloignés de la mer. Il est vrai que l'on ne s'explique pas davantage la raison pour laquelle les Grecs de Marseille allaient chercher dans les Maures le dégraissant de leurs innombrables amphores (Reille, 1985; Reille, Abbas, 1992).

L'absence de modèle grec à trémie dans les niveaux lattois du IV s. avant J.-C. apparait comme un argument plus sérieux à opposer à l'hypothèse d'une production directe par les Grecs à cette époque.

\section{Un centre d'obédience indigène?}

À l'inverse, on pourrait imaginer que le centre exploitant les basaltes de Saint-Thibéry/Bessan était, au IVe s., un centre strictement indigène qui produisait essentiellement des meules traditionnelles de type à va-et-vient (fig. $4 \mathrm{~d}$ à g) et quelques modèles novateurs du type rotatif, d'inspiration ibérique.

Sur le plan typologique, ces derniers modèles sont effectivement représentés par des exemplaires à meta épaisse, plano-convexe, et catillus biconcave dénué de bandeau périphérique sur le sommet (fig. 4b). Parmi ces modèles, on a trouvé à Lattes un demi-catillus comportant une "oreille" latérale (fig. 4c). Ces formes spécifiques, spécialement la dernière, sont caractéristiques des meules rotatives anciennes d'Espagne (Alonso-Martinez, 1999 , p. 252 et 254), mais aussi d'Ensérune en Languedoc (Jannoray, 1955). Enfin, le rattachement du centre de Saint-Thibéry/Bessan au monde ibérolanguedocien expliquerait qu'il n'ait pas produit de modèle à trémie de type grec.

Il serait cependant irréaliste de soutenir l'option d'un centre strictement indigène qui tournerait économiquement le dos à un voisin immédiat aussi puissant que la colonie grecque d'Agde, située par surcroît au débouché de la vallée de l'Hérault, axe principal de communication terrestre dans le secteur. 

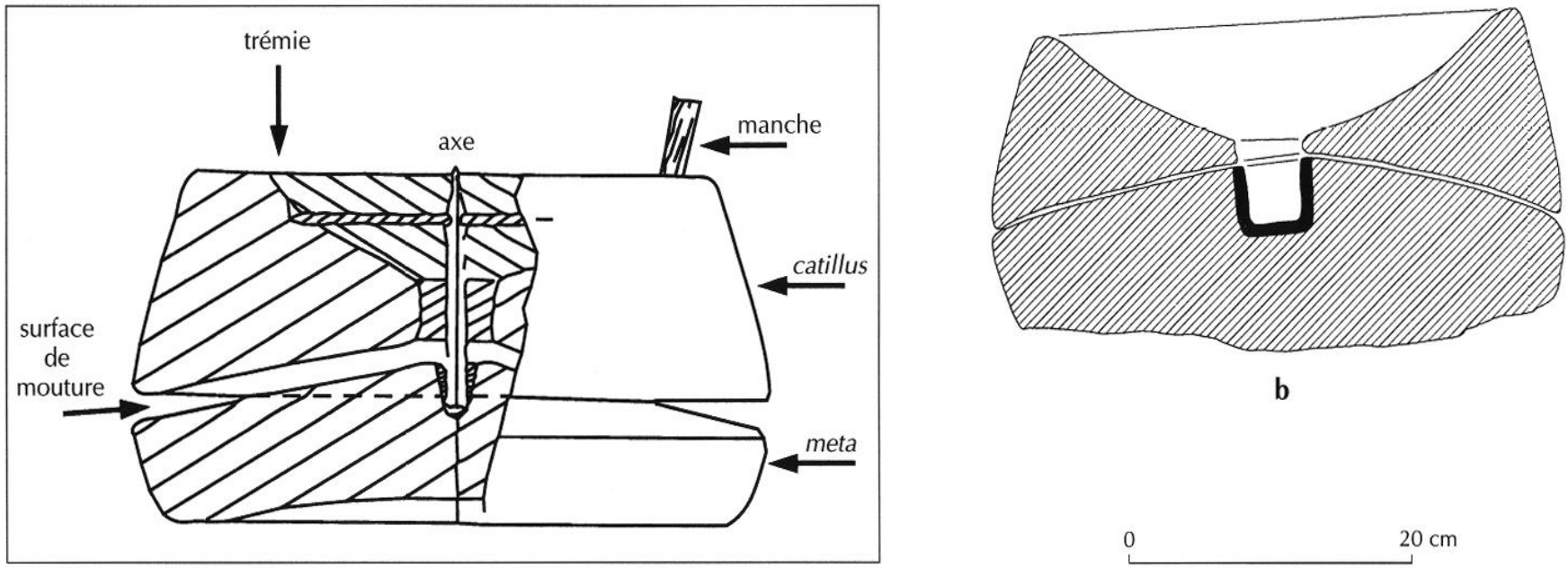

a

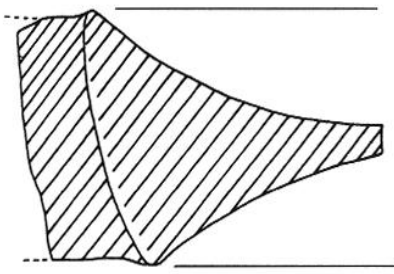

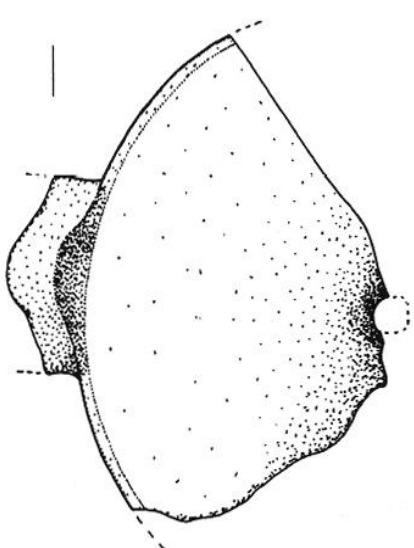

C

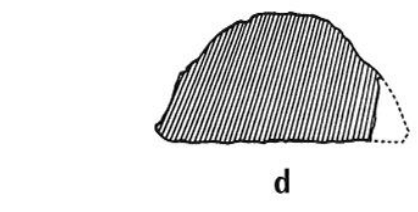

d
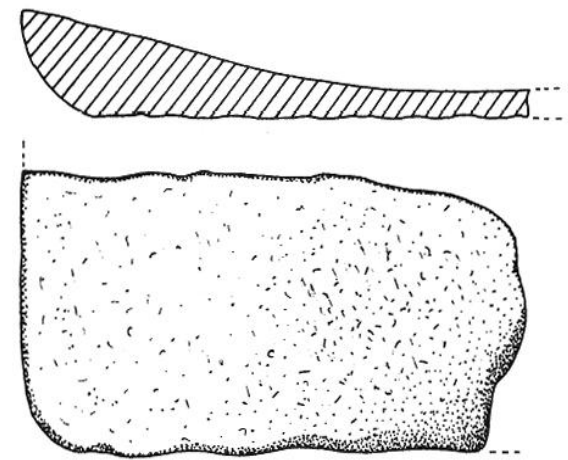

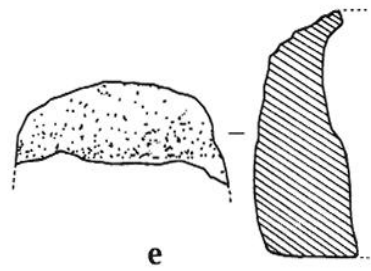

Fig. 4 - a, schéma théorique représentant les éléments constitutifs d'une meule rotative basse d'époque protohistorique ou antique (dessin extrait d'Amouric, 1997, p. 39, fig. IC) ; b-g, représentations de meules laltoises du IV's. avant J.-C. (dessins extraits de Raux, 1999) : b, meule rotative complète, de type ibérique, en basalte de Saint-Thibéry/Bessan, $1^{e r}$ quart du $V^{E}$ s. avant J.-C. (US 27253) ; c, partie de catillus de meule rotative, de type ibérique, avec oreille latérale, en basalte de Saint-Thibéry/Bessan, $2^{2}$ quart du IV s. avant J.-C. (US 27186); d, e, fragments de molettes de système à va-et-vient, en basalte de Saint-Thibéry/Bessan, respectivement $3^{2}$ et $1^{\text {er }}$ quart du $N^{*}$ s. avant.J.-C. (US 1549 et 27245) ;f, g, tables de système à va-et-vient, en basalte de Saint-Thibéry/Bessan, respectivement $1^{\text {er }}$ et $2^{2}$ quart du IV s. avant J.-C. (US 24013 et 27184).

Un centre de tradition technologique indigène bénéficiant du dynamisme économique de la colonie agathoise ?

Cette troisième hypothèse a l'avantage de concilier les résultats des différentes observations.
Sur le plan strictement technico-culturel, il semble bien que l'hypothétique centre de Saint-Thibéry/Bessan soit enraciné dans la culture ibéro-languedocienne. Des meules à va-et-vient portant sa signature et extraites de niveaux antérieurs au IV $\mathrm{IV}^{\mathrm{s}} \mathrm{s}$. apportent la preuve de l'ancienneté de sa production (Reille, 
2000a) ${ }^{3}$. Les remarques précédentes concernant les caractères typologiques des modèles rotatifs vont également dans ce sens. On sait d'autre part que les trois premiers quarts du $\mathrm{V}^{\mathrm{e}} \mathrm{s}$. avant J.C. sont caractérisés localement par une phase importante de développement de la culture ibéro-languedocienne et des échanges avec le monde ibérique (Nickels, 1989; Garcia, 1995b, p. 146). Il est cohérent d'admettre que c'est à travers cette tradition culturelle que le modèle rotatif a été originellement diffusé.

Sur le plan économique et commercial, il parait vraisemblable que la colonie agathoise, alors en pleine expansion, ait joué un rôle important dans la diffusion de la production saint-thibérienne.

L'exportation par voie maritime vers Lattes ou vers le nord dans la vallée de l'Hérault et par voie terrestre atteste la vigueur de cette diffusion : exemplaires rotatifs de l'oppidum de La Ramasse (Garcia, 1993 ; Reille, 1995).

Elle se poursuit encore aux II' et Ir s. avant J.-C.., alors même que les meules rotatives basses et les meules à trémie du Cap d'Agde, produites quasi industriellement, submergent le Languedoc oriental et la Provence ${ }^{4}$. On rencontre alors les productions de Saint-Thibéry/Bessan jusqu'à Martigues et Aix-en-Provence, bien qu'en proportions plus modestes et avec une typologie conforme aux canons de l'époque (Nin, 1991 ; Chausserie-Laprée, 1998 ; Reille, 1998, 2000b) ${ }^{5}$.

\section{Le site du Cap d'Agde}

Comparativement, au IV ${ }^{e}$ s. avant J.-C., le site côtier d'Embonne/Cap d'Agde paraît bien discret sur le plan des exportations, en dépit de la supériorité incontestable de sa position géographique pour le commerce maritime.

Sa production, modestement représentée à Lattes, comporte des modèles traditionnels du type à va-et-vient ainsi que des modèles rotatifs, mais apparemment plus

3. Des résultats récents, en instance de publication, montrent que les meules basaltiques de Saint-Thibéry/Bessan faisaient l'objet d'exportations relativement lointaines dès l'âge du Bronze.

4. Comme le souligne justement D. Garcia (1995b, p. 158), le rôle d'Agde dans la diffusion de la meule rotative basse a effectivement été essentiel.

5. Des observations récentes nous permettent d'étendre jusqu'à Olbia/Hyères la zone de diffusion orientale des meules rotatives tardives de Saint-Thibéry/Bessan (résultats en instance de publication). tardifs d'un demi-siècle que ceux de Saint-Thibéry/ Bessan. D'autre part, aucun exemplaire grec à trémie originaire du Cap d'Agde n'a encore été exhumé dans les niveaux lattois du IV's. avant J.-C.

Il est possible que son statut ait été à l'époque assez proche de celui que nous avons envisagé pour Saint-Thibéry/Bessan. Centre de tradition indigène, il fournissait déjà des meules à va-et-vient antérieurement au IV ${ }^{\text {e }} \mathrm{s} .{ }^{6}$. Il a vraisemblablement bénéficié, lui aussi, du remarquable instrument de diffusion commerciale détenu par la colonie grecque. La déficience relative perçue à Lattes est peut-être le reflet d'une capacité de production alors nettement inférieure à celle du centre saint-thibérien ? Au IVe s., l'époque de l'exploitation intensive était encore à venir pour Embonne/Cap d'Agde. L'étude récente des meules de Pech Maho montre par ailleurs que cette situation s'est vraisemblablement perpétuée pendant le III $^{\mathrm{e}} \mathrm{s}$., ce qui conduirait à situer le début des exportations massives vers le commencement du II $^{\mathrm{e}}$ s. avant J.-C. (Reille, 2000a).

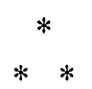

L'analyse pétrographique microtexturale appliquée aux meules du site portuaire de Lattes, l'un des plus proches d'Agde, nous conduit aux constatations et interprétations suivantes.

La prééminence du centre de production d'Embonne/Cap d'Agde dans la distribution des meules à grain, prééminence attestée à partir du $\mathrm{II}^{\mathrm{c}} \mathrm{s}$. avant J.-C. en Languedoc oriental et en Provence, apparaît comme inexistante au niveau des importations lattoises pendant toute la durée du IV $\mathbf{s}$. avant J.-C.

À cette époque, ces importations étaient en effet largement dominées par les productions d'un hypothétique centre de fabrication, vraisemblablement situé dans l'arrière-pays agathois, à proximité du volcan de SaintThibéry/Bessan.

La signature microtexturale typique du basalte de Saint-Thibéry/Bessan s'observe notamment dans les toutes premières meules rotatives distribuées en Languedoc oriental (premier quart du IVe s. avant J.-C.). Cette signature se retrouve également dans des meules d'époque tardive (II -I Ir $^{\text {er }}$. avant J.-C.), mêlées à la très abondante production du Cap d'Agde, comme témoin

6. Matériel en cours d'étude. 
d'une activité d'extraction qui s'est obstinément poursuivie pendant au moins trois siècles sur un site de l'arrièrepays.

Dans l'état actuel des connaissances, sur le plan technico-culturel, on peut supposer que le centre de Saint-Thibéry/Bessan était enraciné dans la tradition ibéro-languedocienne. Il est également vraisemblable

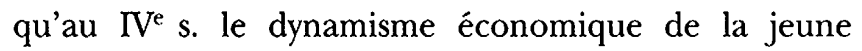
colonie agathoise, alors en pleine expansion, ait joué un rôle déterminant dans la diffusion de sa production.

En allait-il de même, à cette époque, pour le futur " gisement-vedette " d'Embonne/Cap d'Agde? Sa représentation statistiquement déficiente dans la proche cité de Lattes comme l'absence de meules à trémie de type grec parmi les témoins actuellement connus de sa production ancienne permettent de poser la question.
En Gaule méridionale, pour apprécier les modalités de diffusion de l'importante innovation technique que constitue la meule à mouvement rotatif, il n'est pas indifférent de constater le rôle précoce et notable joué par un centre de production, jusque-là insoupçonné, dont l'enracinement dans la culture technique ibérolanguedocienne peut être sérieusement envisagé ?

7. Je remercie collectivement tous ceux qui m'ont aidé dans ce travail, notamment M. Py et l'ensemble de l'équipe de Lattes, plus spécialement S. Raux; G. Marchand, proche collaborateur du regretté A. Nickels, qui m'a fait part d'utiles précisions concernant leurs travaux; D. Garcia pour l'aide précieuse qu'il apporte constamment à notre démarche et pour les échanges amicaux et passionnés sur l'appartenance culturelle du centre hypothétique de production de meules de Saint-Thibéry/Bessan; P. Arcelin qui a bien voulu examiner le manuscrit et me faire bénéficier de ses suggestions et remarques. Ce travail a, notamment, reçu l'aide financière du Service régional de l'archéologie de Provence-Alpes-Côte d'Azur et de l'association AssTerr. 


\section{BIBLIOGRAPHIE}

Ai.oNSO-MARTINE\% N.

1995 : Les premières meules rotatives dans le nord-est de la péninsule Ibérique, in: Tables rondes d'Aix-enProvence, avril 1993-mai 1994, Cahier d'histoire des techniques, 3, Publications de l'université de Provence, p. 15-24.

1997 : Origen y expansion del molino rotativo bajo en el mediterraneo occidental, in: Garcia D., Meeks D. (éds), Techniques et économie antiques et médiévales. Le temps de l'innovation, Paris, éd. Errance, coll. Archéologie aujourd'hui, p. 15-19.

1999 : De la llavor a la farina. Els processos agricoles protohistorics a la Catalunya occidental, Monographies d'archéologie méditerranéenne, 4, Lattes, $328 \mathrm{p}$.

\section{AMOURETTI M.-C.}

1986 : Le pain et l'huile dans la Grèce antique, évolution des techniques agraires d'Hésiode à Théophraste, Besançon, Centre de recherches d'histoire ancienne de Besançon, 67, $330 \mathrm{p}$.

1997 : Introduction, in: Garcia D., Meeks D. (éds), Techniques et économie antiques et médiévales. Le temps de l'innovation, Paris, éd. Errance, coll. Archéologie aujourd'hui, p. 7-12.

Amouric: $\mathrm{H}$.

1997 : L'anille et les meules, in: Garcia D., Meeks D. (éds), Techniques et économie antiques et médiévales. Le temps de l'innovation, Paris, éd. Errance, coll. Archéologie aujourd'hui, p. 39-47.

ARIS R.

1963 : L'industrie du basalte dans l'Antiquité à Agde, les fabriques de meules, les carrières et la ville légendaire d'Embonne, Bulletin de la fédération historique du Languedoc méditerranéen et du Roussillon, p. 129-135.

1974 : Le site préromain d'Embonne: une antique fabrique de meules sous la nouvelle ville du Cap-d'Agde, Études sur Pézenas et sur l'Hérault, 5, 1, p. 3-18.

1986-1987: Notes sur l'histoire d'Agde, Études sur Pézenas et sur l'Hérault, n.s., 2-3, p. 1-18.

BotTIN C.

1905 : Rapport sur la découverte de deux meules gallo-romaines par M. le Colonel Noir. Étude historique de l'atelier de ces meules situé sur le plateau du Rocher de l'Aigle et à la Guérarde, extraits des Annales de l'Académie du Var, année 1905.

BRUN J.-P.

1997 : L'introduction des moulins dans les huileries antiques, in: Garcia D., Meeks D. (éds), Techniques el économie antiques et médiévales. Le temps de l'innovation, Paris, éd. Errance, coll. Archéologie aujourd'hui, p. 69-78.

ChaUSSERIE-LAPRÉE J.

1998 : Les meules des habitats protohistoriques de Martigues, Documents d'archéologie méridionale, 21, p. 211-235.

Coulouma J., Claustres G.

1943 : L'oppidum de Cessero près de Saint-Thibéry (Hérault), Gallia, I, 2, p. 1-18.

DAUTRIA J.-M., LIOTARD J.-M.

1990 : Les basaltes d'affinité tholéiitique de la marge méditerranéenne française, Comptes rendus de l'Académie des Sciences de Paris, 311, série II, p. 821-827.

Dautria J.-M., ReIlle J.-L.

1992 : Analyse pétrographique et détermination de la provenance du matériau basaltique des meules antiques de Lattes, Lattara, 5, p. 233-236.

Domergue C., Béziat D., Cauuht B., JaRrier C., I LANDFS C., Morast J.-G., Oliva P., Pulou R., Tollon F.
1997 : Les moulins rotatifs dans les mines et les centres métallurgiques antiques, in: Garcia D., Meeks D. (éds), Techniques el économie antiques et médiévales. Le temps de linnovation, Paris, éd. Errance, coll. Archéologie aujourd'hui, p. 48-61.

\section{GaRCIA D.}

1993 : Entre Ibères et Ligures. Lodévois et moyenne vallée de l'Hérault protohistorique, suppl. 26 à la Revue archéologique de Narbonnaise, Paris, CNRS Éditions, 355 p.

1995a : I.es carrières de meules d'Agde grecque et la diffusion du type rotatif en Gaule méridionale, in: Tables rondes d'Aix-en-Provence, avril 1993-mai 1994, Cahier d'histoire des techniques, 3 , Publications de l'université de Provence, p. 25-32.

1995b : Le territoire d'Agde grecque et l'occupation du sol en Languedoc central durant l'Âgc du Fer, in: Sur les pas des Grecs en Occident. Hommages à André Nickels, Études massaliètes, 4, p. 137-168.

Garcia D., Meeks D. (íns)

1997 : Techniques et économie antiques et médiévales. Le temps de l'innovation, Paris, éd. Errance, coll. Archéologie aujourd'hui, $240 \mathrm{p}$.

JANNORAY J.

1955 : Ensérune. Contribution à l'étude des civilisations préromaines de la Gaule méridionale, Bibliothèques des Écoles françaises d'Athènes et de Rome, 181, Paris, De Boccard, 490 p. et LXXI pl. h. t.

JULLY J.J.

1983 : Céramiques grecques ou de type grec et autres céramiques en Languedoc méditerranéen, Roussillon et Catalogne, partie 2, Paris, Les Belles Lettres, 1561 p. [p. 1203 et 1 323-1 333].

I AAYET J.

1950 : Le livre de La Courtine (ville 
antique de la région toulonnnaise), Archéologie protohistorique, Toulon, ouvrage publié avec le concours du CNRS, p. 37-103 et $111-155$.

LefìvRE C., DupuY C.

1972 : Sur la présence de basaltes à hypersthène normatif dans les volcans du Bas-Languedoc, Comptes rendus de l'Académie des Sciences de Paris, 274, série D, p. 2 616-2 619.

\section{MusÉE GRANET (OUVRAGE COLLECTIF)}

1987 : Archéologie d'Entremont au musée Granet, Aix-en-Provence, musée Granet, 267 p.

\section{NICKELS A.}

1976 : Contribution des fouilles de l'arrière-pays d'Agde à l'étude du problème des rapports entre Grecs et indigènes en Languedoc ( $\mathrm{VI}^{\mathrm{c}}$ $\mathrm{V}^{\mathrm{e}}$ siècles), Mélanges des Écoles françaises de Rome et d'Athènes, 88, 1, p. 141-157.

1987 : Le site protohistorique du Mont Joui à Florensac (Hérault), Revue archéologique de Narbonnaise, 20, p. 3-42.

1989 : La Monédière à Bessan (Hérault). Le bilan des recherches, Documents d'archéologie méridionale, 12, p. 51-120.

1995 (p.m.): Les sondages de la rue Perben à Agde (Hérault), in: Sur les pas des Crecs en Occident. Hommages à André Nickels, Études massaliètes, 4, p. 59-98.

NIN N.

1991 : Archéologie et développement urbain. En préliminaire à l'opération Sextius-Mirabcau: lcs recherches archéologiques réalisées sur le terrain Coq, Impressions du musée Cranet, Association des amis du musée Granet, 6, p. 30-35.

\section{Peacock D. P. S}

1986 : The production of roman millstones near Orvieto, Umbria, Italy, The Antiquaries Journal, 66, p. 45-51.

\section{PY M.}

1992 : Meules d'époque protohistorique et romaine provenant de Lattes, Lattara, 5, p. 183-232.

PY M., GARCIA D.

1993 : Bilan des recherches archéologiques sur la ville portuaire de Lattara (Lattes, Hérault), Gallia, 50, p. 1-93.

Raux S.

1999 : Les objets de la vie quotidienne à Lattes, au IV ${ }^{\mathrm{e}}$ s. av. J.-C., Lattara, 12, p. $439-518$.

REIL.LE J.-L.

1985 : L'analyse pétrographique des céramiques et le problème de la provenance des amphores massaliètes (IV'-II ${ }^{\mathrm{e}}$ s. av. J.-C.), Documents d'archéologie méridionale, 8, p. 101-112.

1995 : La diffusion des meules dans la vallée de l'Hérault à l'époque protohistorique, et l'identification microtexturale des basaltes, Documents d'archéologie méridionale, 18, p. 197-205.

1998 : L'importation des meules en basalte dans le secteur de Martigues au deuxième Âge du Fer : identification pétrographique des sources, Documents d'archéologie méridionale, 21, p. 237-244.
1999 : Détermination pétrographique de l'origine des meules de Lattes au $I^{e}$ s. av. n. è. Changements et contrastes dans les importations, Lattara, 12, p. 519-522.

2000a: L'importation des meules à grains en Languedoc occidental au deuxième Âge du Fer sur les sites de Pech Maho (III ${ }^{\mathrm{e}}$ s. av.) et Cayla de Mailhac (V ${ }^{\mathrm{e}}$ au II ${ }^{\mathrm{e}} \mathrm{s}$. av.), Documents d'archéologie méridionale, 23, p. 273-278.

2000b : Agde et le commerce des meules à grains en Gaule méditerranéenne à la fin de l'Âge du Fer (II ${ }^{\text {- }}$ Ir $^{\text {er }}$ siècles av. n. è.), in: Buxo R. I., Pons E. (éds), Els productes alimentaris d'origen vegetal a l'edat del ferro a l'Europa occidental : de la producció al consum, sèri monográfica del museu d'arqueologia de Catalunya-Girona, Comptes rendus du XXII ${ }^{e}$ colloque international pour l'étude de l'Âge du Fer, Girona, 21-24 mai 1998, p. 359-364.

\section{REILle J.-L., AbBas G.}

1992 : Les inclusions minérales des amphores massaliètes et leur signification : le cas des formes archaiques et le problème de la localisation des sites de production, Documents d'archéologie méridionale, 15, p. 431-437.

\section{ReILle J.-L., Chabot L.}

2000 : L'origine et l'importation des meules à grains dans un village de la chôra marseillaise aux II $^{e}$ et $I^{\text {er }}$ s. av. n. è. (oppidum de la Cloche, Les Pennes-Mirabeau, B.-du-Rh.), Documents d'archéologie méridionale, 23, p. 279-282. 